\title{
Terahertz-optical properties of a bismuth ferrite single crystal
}

\author{
M. Białek, ${ }^{1, *}$ T. Ito, ${ }^{2} \mathrm{H}$. Rønnow, ${ }^{1}$ and J.-Ph. Ansermet ${ }^{1}$ \\ ${ }^{1}$ Institute of Physics, Ecole Polytechnique Fédérale de Lausanne (EPFL), 1015 Lausanne, Switzerland \\ ${ }^{2}$ Electronics and Photonics Research Institute, National Institute of Advanced Industrial Science and Technology (AIST), \\ Tsukuba, Ibaraki 305-8565, Japan
}

(Received 30 November 2018; published 21 February 2019)

\begin{abstract}
Transmission through a (100)-oriented single crystal of bismuth ferrite $\left(\mathrm{BiFeO}_{3}\right)$ was studied at frequencies of $0.485-0.765 \mathrm{THz}$ with a continuous-wave source and in the temperature range of $30-350 \mathrm{~K}$ as a function of polarization angle. The fine splitting of spin-wave modes that could be detected with our vector network analyzer-based spectrometer is shown to depend on polarization angle. The crystal shows strong, temperaturedependent, linear dichroism. The interference pattern produced in the sample slab revealed a huge birefringence. The fine splitting dependence on polarization angle suggests correlation with the observed dichroism and the birefringence.
\end{abstract}

DOI: 10.1103/PhysRevB.99.064429

\section{INTRODUCTION}

Bismuth ferrite $\mathrm{BiFeO}_{3}$ (BFO) is an interesting research target as a room temperature multiferroic material with antiferromagnetic and ferroelectric orders [1] and a magnetoelectric coupling [2-5]. The Néel temperature of bismuth ferrite is $643 \mathrm{~K}$, and the ferroelectric Curie temperature is about $1100 \mathrm{~K}$ [6]. A crystal of bismuth ferrite is a rhombohedrally distorted perovskite, where iron atoms form a monoclinic sublattice [7]. The direction of the electrical polarization $\mathbf{P}$ in the crystal is along the pseudocubic (111) direction. Spins are oriented antiferromagnetically between adjacent haxagonal layers of iron atoms. The Dzyaloshinskii-Moriya interaction (DM) [8] is rotating the antiferromagnetic vector forming a spin cycloid with the period of $62 \mathrm{~nm}$ [9].

Two families of spin waves are distinguished: the $\Phi$ modes where spins oscillate in the plane of the cycloid, and the $\Psi$ modes where they oscillate out of this plane. The periodic spin structure folds spin-wave bands, which allows to optically excite higher frequency modes of spin waves [10]. A more detailed investigation shows that these modes are split due to anharmonicity of the spin cycloid distortion, caused by a magnetic anisotropy and another DM interaction [11]. Most notably, the strong $\Psi_{1}$ resonance is split into two modes: $\Psi_{1}^{1}$ and $\Psi_{1}^{2}$. In our previous work, we have shown, that in a polycrystaline sample, this splitting is changing with temperature, because of the temperature dependence of the magnetic anisotropy [12].

As a ferroelectric, BFO is known to show quite a strong and temperature-dependent birefringence. The birefringence

\footnotetext{
*marcin.bialek@epfl.ch
}

Published by the American Physical Society under the terms of the Creative Commons Attribution 4.0 International license. Further distribution of this work must maintain attribution to the author(s) and the published article's title, journal citation, and DOI. is uniaxial, with $\Delta n=n_{e}-n_{o} \approx-0.093$ at $0 \mathrm{~K}$ at a visible wavelength [13]. The optical axis of the crystal (OA) is parallel to the ferroelectric polarization $\mathbf{P}$. The optical birefringence in $\mathrm{BFO}$ is negative, so that the extraordinary refractive index $n_{e}$, for polarization along $\mathbf{P}$, is smaller than the ordinary refractive index $n_{o}$. The optical birefrigence slowly drops with temperature, and, since its origin is from both magnetic and ferroelectric orders, it does not disappear above the Néel temperature [13]. At terahertz $(\mathrm{THz})$ frequencies birefringence was observed in a number of anisotropic materials, some of which, show huge values of $\Delta n$, for example, 3.3 for rutile at $1 \mathrm{THz}$ [14], 1.8 for $\mathrm{LiNbO}_{3}$ also at $1 \mathrm{THz}$ [15] or 1.5 for lead germante $\mathrm{Pb}_{5} \mathrm{Ge}_{3} \mathrm{O}_{11}$ at $0.5 \mathrm{THz}$ [16].

Dichroism results usually from a crystalline anisotropy. However, electric and magnetic orders might induce additional dichroism. The magnetic anisotropy gives rise to the magnetic linear dichroism $[17,18]$ and the ferroelectricty gives rise to the ferroelectric dichroism [19]. Likewise, the $\mathrm{x}$-ray linear dichroism is an established method used to identify ferroelectric and magnetic domains in BFO [3]. At sub$\mathrm{THz}$ frequencies, the broadband absorption originates from broadening and softening of phonon modes with temperature $[16,20,21]$. There are 18 optical phonon modes in BFO: 4 in the $A_{1}$ family infrared active along $\mathbf{P}$, nine in the $E$ family infrared active in the plane perpendicular to $\mathbf{P}$ and five infrared silent modes in the $A_{2}$ family [22]. Directional dichroism (optical diode effect) was shown in $\mathrm{BFO}$ at $\mathrm{THz}$ frequencies of spin-wave modes [23]. In Ref. [24], it was shown that a BFO crystal rectifies optical pulses by modulation of the ferroelectric polarization $\mathbf{P}$ and that transmission and reflection depend on the light polarization angle. The highest $\mathrm{THz}$ output was acquired when the polarization was along the ferroelectric vector.

Spin-wave resonances in $\mathrm{BiFeO}_{3}$ were investigated using different $\mathrm{THz}$ spectroscopy techniques [5,23,25-31]. In some of those studies the dependence on polarization angle was examined. In Refs. [23,28,29], it was shown that the amplitudes of spin-wave resonances in a 001-oriented single crystal were 

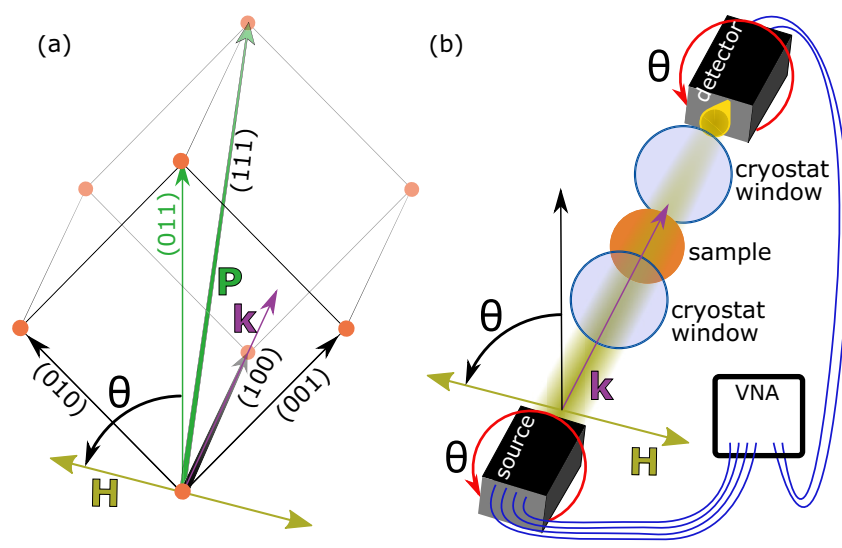

FIG. 1. (a) Iron-sublattice cell with the ferroelectric polarization $\mathbf{P}$ along (111) direction. The wave vector of radiation $\mathbf{k}$ in the experiment is along (100) direction. The angle $\theta$ is defined as the angle between $\mathbf{H}$ field of the electromagnetic radiation and the (011) direction. (b) The $\mathrm{THz}$ radiation was entering the cryostat though sapphire windows. The rotation of polarization was achived by a parallel rotation of the source and the detector, where angle $\theta$ was the angle between vertical axis and a $\mathrm{THz} \mathbf{H}$ field.

larger for polarization perpendicular to $\mathbf{P}$. This observation was explained in terms of the symmetry of the spin-wave excitations of the spin cycloid [11,32]. However, in Ref. [29] the authors argued that the dominance of one magnetic domain of the crystal is necessary to fully explain these results. The angular dependence of these resonances was investigated in detail in Ref. [33], where authors have concluded, contrary to Ref. [29], that the amplitude of spin-wave modes is largely governed by a magnon coupling to phonons.

Here we report experiments performed with a (100)oriented crystal using a spectrometer that offers high frequency resolution and high dynamic range. We show strong anisotropic optical properties of the crystal-the dichroism and the birefrigence-and how they relate with the newly reported fine-splitting of spin-wave resonances.

\section{EXPERIMENTAL}

We have investigated a single crystal sample produced in AIST, Japan [34]. The sample had dimensions of about $3 \times 3 \times 1 \mathrm{~mm}^{3}$. The largest surface of the sample was (100)oriented. We measured the temperature dependence of transmission of $\mathrm{THz}$ radiation normal to the sample surface for different angles of linearly polarized $\mathrm{THz}$ radiation. We define the angle of the polarization $\theta$, as the angle between the magnetic field of the incident radiation $\mathbf{H}$ and the $(011)$ direction [Fig. 1(a)], which is along the projection of the electrical polarization $\mathbf{P}$ on the (100) plane. Therefore, at the angle $\theta=0^{\circ}$, the magnetic field $\mathbf{H}$ of the incident radiation is in the plane defined by $\mathbf{P}$ and the wave vector $\mathbf{k}$. At angles $\theta= \pm 90^{\circ}$ the magnetic field $\mathbf{H}$ is perpendicular to $\mathbf{P}$ and the electric field $\mathbf{E}$ is acting along $\mathbf{P}$. During the experiment, we have measured the transmission for polarization angles from $\theta=90^{\circ}$ to $\theta=-90^{\circ}$ with steps of $10^{\circ}$.

The experimental setup uses a vector network analyzer (VNA) and Virginia Diodes frequency extenders in the range of $f=0.485-0.765 \mathrm{THz}$. This setup was used in our laboratory for the investigation of resonances in BFO at low [35] and at high temperatures [12] in polycrystaline samples. The source extender produces $\mathrm{THz}$ frequencies emitted by a corrugated horn into a free beam propagating in air. The frequency extenders naturally operate in the linear polarization defined by the geometry of their rectangular waveguides. Rotation of the polarization was achieved by a parallel rotation of both extenders with respect to the sample.

The schematic of the experimental setup is presented in Fig. 1(b). A copper sample holder had two oversized $(8 \mathrm{~mm}$ in diameter) metallic waveguides which guided the radiation trough the sample. The sample holder was mounted on top of a coldfinger and enclosed in a cryostat with sapphire windows. The sample space was pumped with a turbomolecular pump. We report transmission spectra obtained during warming-up of the sample in the cryostat, when the cold finger compressor was not running. This procedure reduced noise originating from vibrations of the coldfinger and was slow enough to allow for measurements. A typical warm-up of the sample from 30 to $350 \mathrm{~K}$ took $12-14$ hours. Temperature was rising faster at lower temperatures, and above about $200 \mathrm{~K}$ the temperature sweep was linearized using a heater mounted on top of the coldfinger and controlled with a PID. Temperature was measured using two K-type thermocouples positioned at opposite corners of the sample with coldjunctions placed in liquid nitrogen outside the cryostat. Two temperature measurements allowed us to estimate a temperature gradient to be at most $4 \mathrm{~K}$ at the lowest temperatures and strongly decreasing at higher temperatures. We assume this value to be a maximum systematic error of our temperature measurements.

The radiation, which passes the sample, is coupled to a waveguide by another corrugated horn. The detector extender measures the amplitude and the phase of the electric field of the incoming radiation. After nomalization to the source output, this is the $S_{21}(T, f, \theta)$ signal, which in our experiment is the amplitude of transmission. We also collected reflection signals in the $S_{11}$ channel, but we found these data hard to analyze, due to a large portion of reflection that is not affected by the sample. In Sec. III A regarding the dichroism, we report these values in $\mathrm{dB}$ units. In further sections, we show derivatives of transmission $S_{21}$ to temperature $T$

$$
\frac{\partial S_{21}}{\partial T}(T, f, \theta)=\frac{S_{21}(T+d T, f, \theta)-S_{21}(T, f, \theta)}{d T}
$$

which we refer to as the temperature-differential spectra or the signal. We use this data-treatment to offset signals depending on sample temperature from interferences in the experimental setup.

\section{RESULTS}

In the first part of this section, we consider the dependence of a broadband transmission on polarization angle at different temperatures. In the second part, we deduce birefringence from interference patterns. In the last part, we describe our findings on the dependence of spin-wave resonances on polarization angle. 


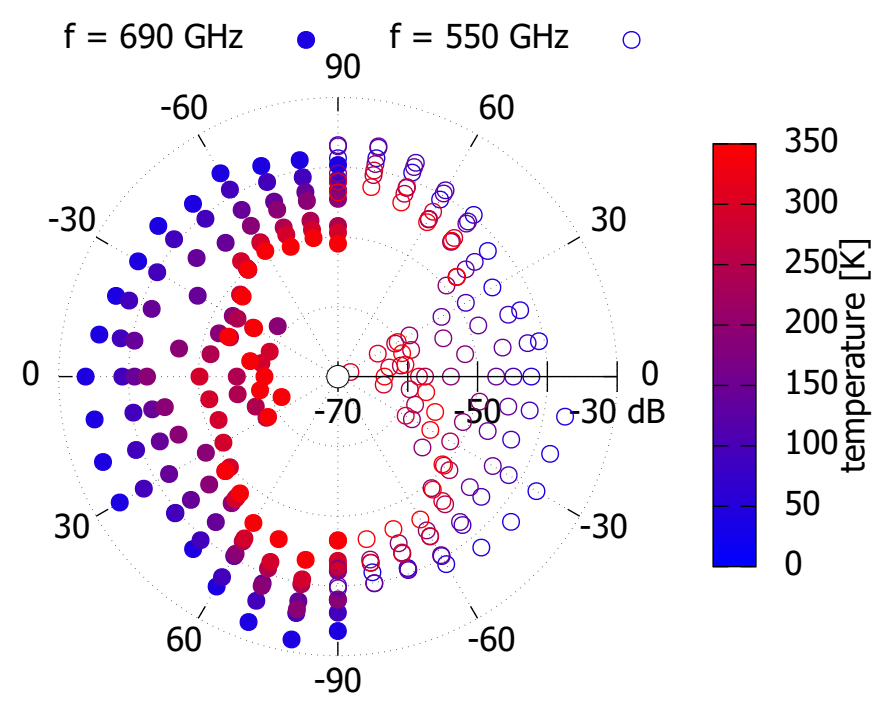

FIG. 2. Dependencies of transmission on the polarization angle $\theta$ in a range of temperatures encoded by a color scale. Data for two selected frequencies: full circles on the left side for $f=690 \mathrm{GHz}$ and open circles on the right side for $f=550 \mathrm{GHz}$.

\section{A. Dichroism}

Transmission $S_{21}(T, f, \theta)$ depends on polarization angle $\theta$ and on temperature $T$ (Fig. 2). At low temperatures, transmission is almost isotropic. With rising temperature, transmission drops much faster for the polarization angle where magnetic field $\mathbf{H}$ is in plane with the electrical polarization $\mathbf{P}\left(\theta=0^{\circ}\right)$. This type of an incident polarization-angle dependence and a huge transmission difference shows that the observed effect is a linear dichroism. We fitted a heurestic function $S_{21}(T, f, \theta)=S_{21}^{90}(T, f)+S_{21}^{d}(T, f) \cos ^{2}\left(\theta-\theta_{0}(T, f)\right)$, where $S_{21}^{90}(T, f)$ is the transmission at $\theta= \pm 90^{\circ}, S_{21}^{d}(T, f)$ is an amplitude of the dichroism and $\theta_{0}(T, f)$ is a possible sample tilt. This transmission versus angle dependence was fitted separately for every frequency-temperature point. A frequency-temperature map of fitted values $S_{21}^{d}(f, T)$ would show interferences and resonances. To focus on the temperature dependence, we averaged fitted values over the entire frequency window for each temperature. These averages, presented in Fig. 3, show that difference of transmission $\left\langle S_{21}^{d}(T)\right\rangle$ in $\mathrm{dB}$ rises approximately lineary with temperature and reaches about $15 \mathrm{~dB}$ at $340 \mathrm{~K}$ (Fig. 3). The fit parameters $\theta_{0}(T, f)$ allowed to estimate the error in the sample positioning to be $-3.3^{\circ}$ with a standard deviation of $8.3^{\circ}$. A similar value of the difference between absorbanaces for perpendicular polarizations was reported for a 001oriented $0.22 \mathrm{~mm}$-thick sample in Ref. [24] $(\approx 14 \mathrm{~dB} / \mathrm{mm})$ at room temperature. Even larger dichroism was reported at room temperature in Ref. [33] for a $0.2 \mathrm{~mm}$-thick sample $(\approx 25 \mathrm{~dB} / \mathrm{mm})$.

The temperature dependence of the dichroism suggests that it cannot be of a magnetic origin. A whole range of different magneto-optical effects show dependencies on either net magnetization or on amplitude of the antiferromagnetic vector [36]. Those drop with rising temperature, so a magnetic origin of the observed dichroism can be ruled out. A similar dependence is expected for a dichroism of a ferroelectric

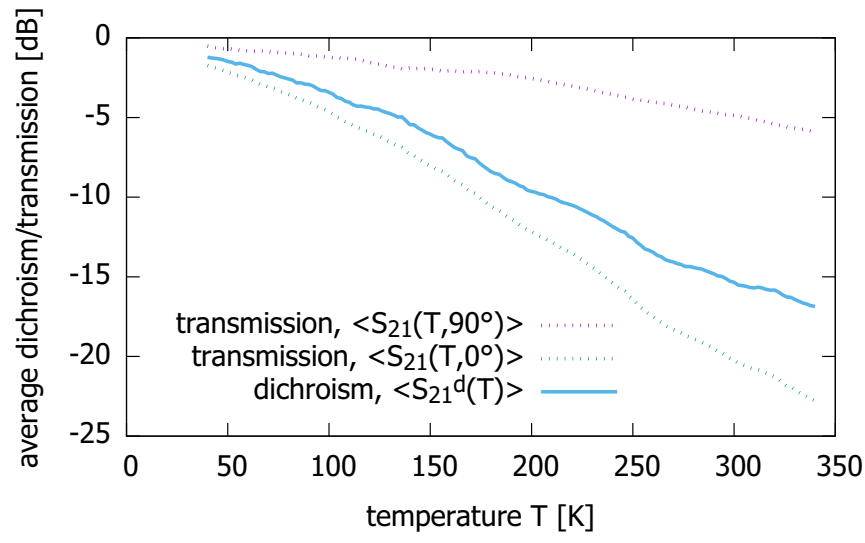

FIG. 3. Frequency-averaged transmission for perpendicular polarizations and the dichroism dependence on temperature.

origin. Therefore, we consider that absorption by phonons is responsible for the observed effect. Transverse phonons of $E$ symmetry in BFO are known to couple more strongly to the radiation with $\mathbf{E}$-field perpendicular to the ferroelectric polarization $\mathbf{P}$ [22,37]. The lowest-frequency $E(1)$ phonon mode has a frequency of about $2 \mathrm{THz}$, so we cannot observe the entire resonant line in our experiment. However, phonons in BFO are known to soften and broaden with increasing temperature [20,22,38-40], so their contribution to the dichroism at our experimental range increases.

\section{B. Birefringence}

To determine the refractive index, we analyzed the interference pattern representing standing waves in the sample slab. This pattern is more clear in temperature-differential transmission (Fig. 4) than in untreated transmission. This is because the refractive index of BFO depends on temperature, while all other interference patterns are almost temperatureindependent. The period of the pattern changes with polarization angle in such a way that they are very similar to either those at $\theta=0^{\circ}$ or $\theta=-90^{\circ}$. For angles in the ranges of about $|\theta|=30^{\circ}-60^{\circ}$, the patterns were not clear (data not shown), because the two interference patterns were superimposed. This observation reflects the fact that there are two rays present in the sample, which change intensity with rotation of the polarization angle. Therefore, in the following analysis, we used the results for $\theta=0^{\circ}$ and $\theta=-90^{\circ}$ presented in Fig. 4. The interference pattern for $\theta=0^{\circ}$ allows us to calculate the refractive index $n_{o}$ for an ordinary ray, since in this situation the electric field of the incoming wave is perpendicular to the optical axis of the crystal (OA). In the case of $\theta=-90^{\circ}$, the electric field is not entirely along the OA, because the latter is not parallel to the sample surface. Nevertheless, if $n_{o}$ is known, it is possible to calculate $n_{e}$, as explained in the following paragraphs.

We calculated the ordinary ray refractive index $n_{o}$ using frequencies of interference pattern maxima [Fig. 4(a)]. We assumed that the sample is a Fabry-Perot cavity and that the refractive index is frequency-independent far from resonances. Thus, we estimated the mean value of the refractive index in the frequency window of 500-750 GHz, outside 
(a) $\theta=0^{\circ}, \mathrm{n}_{0}$

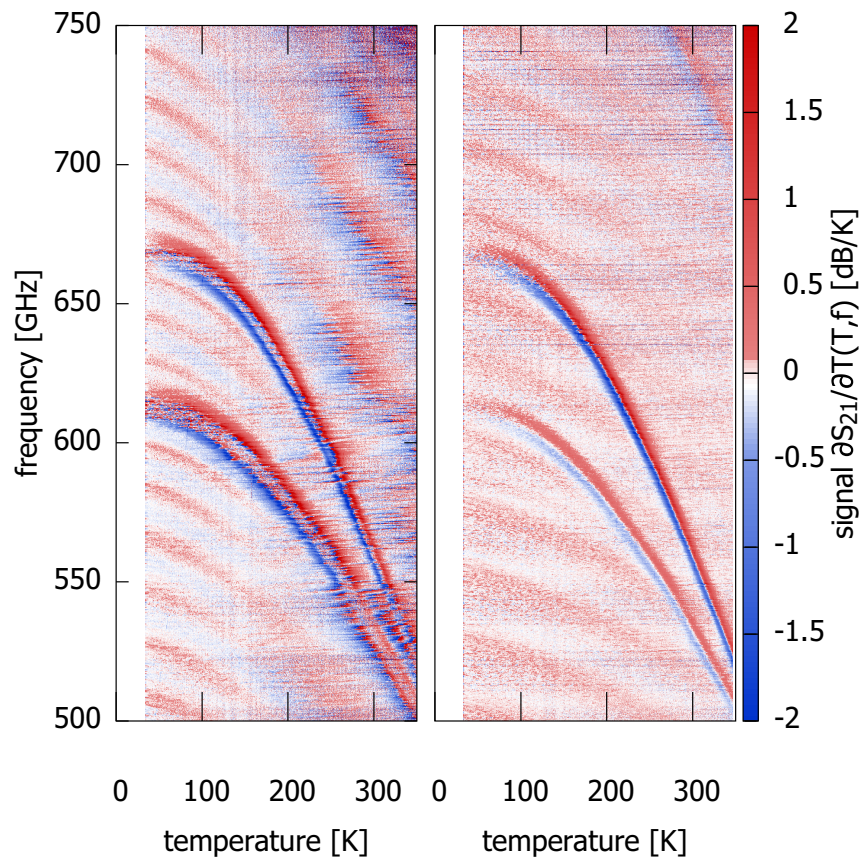

FIG. 4. Temperature-differential spectra for (a) $\mathbf{E}$ field perpendicular to the electrical polarization $\mathbf{P}$ and (b) parallel.

resonances. We found that

$$
n_{o}=\frac{c m}{2 d f_{m}^{o}},
$$

where $d=1.0 \mathrm{~mm}$ is the sample thickness, $c$ the speed of light in vacuum and $f_{m}^{o}(T)$ the frequency of the $m$ th interference maximum. Convergence of $n_{o}$ values [Fig. 5(b)] for all the interference maxima was obtained with the lowest frequency maximum [510 $\mathrm{GHz}$ at $30 \mathrm{~K}$ in Fig. 4(a)] having $m=30$. This assumption sets our estimation of the uncertainty of the refractive index to about 0.1 .

In the temperature range of $130-210 \mathrm{~K}$ the interference period grows by a factor of 3 [Fig. 4(a)]. Since this is
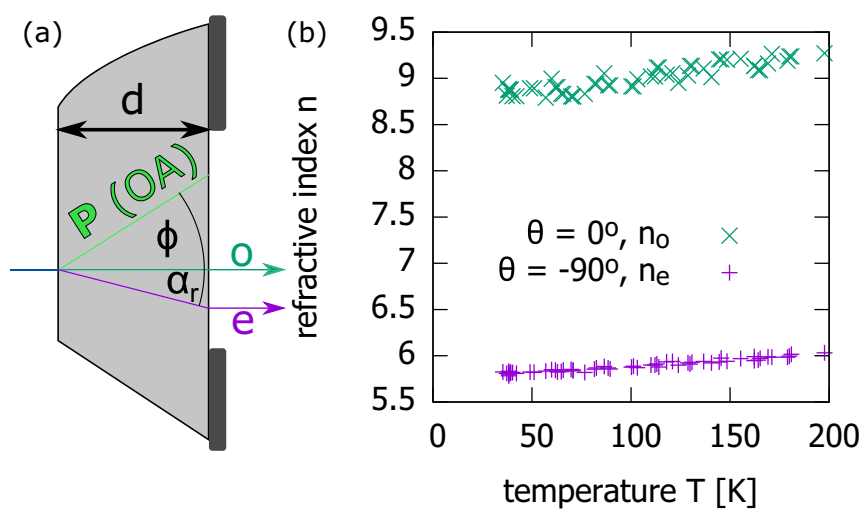

FIG. 5. (a) Schematic cross-section of the sample. Optical axis (OA) was inclined at the angle $\phi$ to the 100 surface normal. Sample was placed over a circular aperture. Traces of the ordinary $(o)$ and the extraordinary $(e)$ rays in the sample are shown. (b) Determined mean refractive indices of $\mathrm{BFO}$ in the $500-750 \mathrm{GHz}$ range. accompanied by a huge growth of sample absorption we do not interpret the high temperature pattern for $\theta=0^{\circ}$ as an interference in the sample slab over a known dimension. This high-temperature interference pattern is highly asymmetric and seems to be strongly dependent on position of the source. Therefore, this pattern might rather reflect electromagnetic modes propagating over the sample surface, bounded by some parts of the sample holder.

Finding refractive index $n_{e}$ of the extraordinary ray is more complex. First, the interference cavity length is longer in the case of the extraordinary ray, because radiation is refracted for the incident angle $\alpha_{i}=0^{\circ}$. The refraction angle $\alpha_{r}$ [Fig. 5(a)] is defined by [41]

$$
\tan \alpha_{r}=\frac{\left(n_{e}^{2}-n_{o}^{2}\right) \cos \phi \sin \phi}{n_{e}^{2} \cos ^{2} \phi+n_{o}^{2} \sin ^{2} \phi},
$$

where $\phi=54^{\circ} 44^{\prime}$ [Fig. 5(a)] is the angle between the ordinary ray propagation direction (100) and the optical axis (111) [1]. Additionally, the refractive index of the extraordinary ray $n_{e}^{\prime}$ depends on the angle $\phi-\alpha_{r}$ between its propagation direction and the optical axis (note that $\alpha_{r}<0$ if $n_{e}<n_{o}$ )

$$
\frac{1}{n_{e}^{\prime}}=\sqrt{\frac{\cos ^{2}\left(\phi-\alpha_{r}\right)}{n_{o}^{2}}+\frac{\sin ^{2}\left(\phi-\alpha_{r}\right)}{n_{e}^{2}}} .
$$

To find $n_{e}$, we solved the equation

$$
n_{e}^{\prime}=\frac{c m}{2 d f_{m}^{e}} \cos \alpha_{r},
$$

where $f_{m}^{e}$ is the frequency of the $m$ th interference maximum [in Fig. 4(b), the maximum at $505 \mathrm{GHz}, 30 \mathrm{~K}$, has $m=20$ ]. We found that the extraordinary refractive index $n_{e}$ grows almost lineary with temperature [Fig. 5(b)]. Since $n_{o}$ is necessary to find $n_{e}$, there are no values for temperatures above $200 \mathrm{~K}$, despite the fact that the interference pattern for the extraordinary ray can be traced up to room temperature. The birefringence grows slowly from $\Delta n \approx-3.0$ at low temperatures to about -3.2 at $200 \mathrm{~K}$, contrary to the dichroism, which grows very strongly with temperature. These values correspond to the extraordinary-ray refraction angle $\alpha_{r} \approx-18.5^{\circ}$.

Similar large difference in a period of an interference pattern was shown in results obtained at room temperature presented in Ref. [33]. Two refractive indices were measured in a (001)-oriented single crystal using time domain spectroscopy in Ref. [24]. Around $600 \mathrm{GHz}$, at room temperature, those were approximately 8.3 for the ordinary ray and 6.6 for the extraordinary ray. While the value for $n_{e}$ is close to a linear extrapolation of our results to room temperature, the value for $n_{o}$ is much smaller than in our result. This might indicate that the sample used in our experiment did not show identical anisotropic properties as the one used in Ref. [24]

\section{Fine splitting of spin-wave modes}

In Fig. 4, two resonant lines can be clearly distinguished as superimposed over a temperature-dependent interference for $\theta= \pm 90^{\circ}$. The identification of modes, as presented in following paragraphs, is based on their frequencies determined by other experimental data [23,28,42]. These modes are, in the order of increasing frequency, $\Psi_{1}^{(2)}$ and $\Psi_{1}^{(1)}$. Data taken 


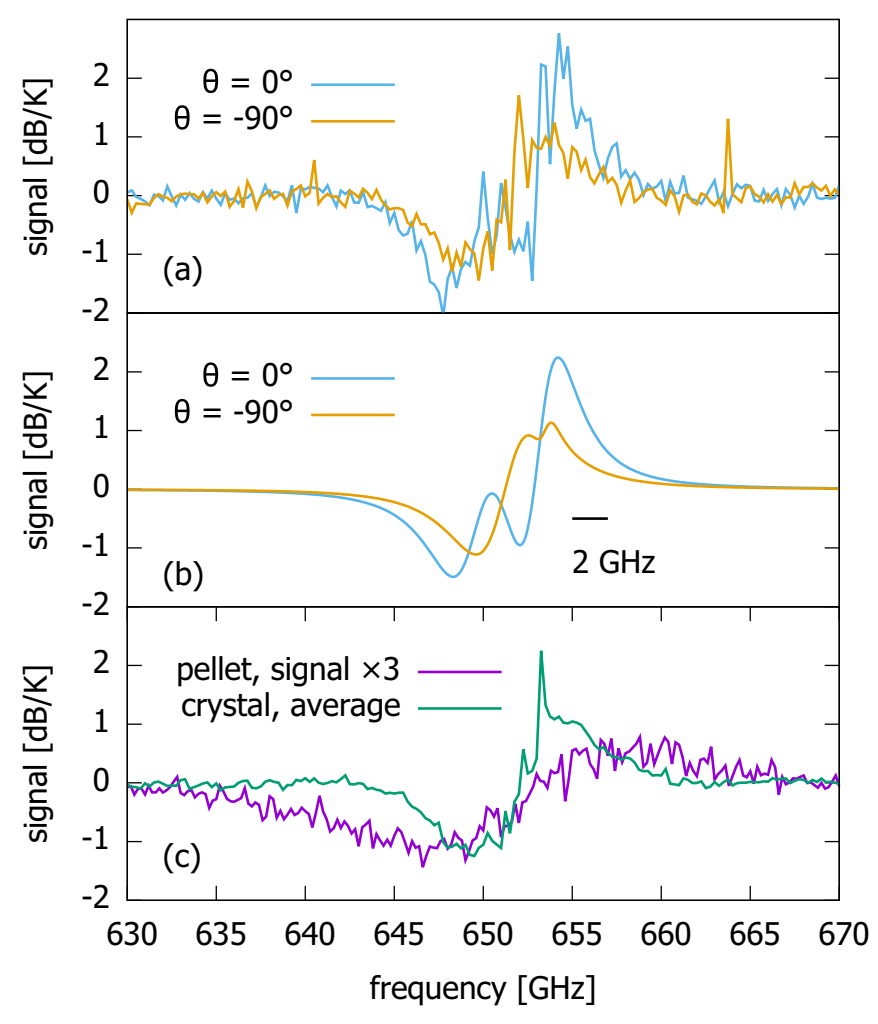

FIG. 6. Comparisons of traces of the $\Psi_{1}^{(1)}$ resonance at $150 \mathrm{~K}$. (a) Resonance for perpendicular polarizations. (b) Respective fits of sums of two derivatives of Lorentzians. (c) Average of results for all angles in the single crystal compared with the resonance trace in the pellet sample.

at $0^{\circ}$ show a splitting of these modes by about $5 \mathrm{GHz}$. In the following discussion, we name these new modes accordingly to their frequencies as $\Psi_{1}^{(2), 1}, \Psi_{1}^{(2), 2}, \Psi_{1}^{(1), 1}$ and $\Psi_{1}^{(1), 2}$. An extract of $\Psi_{1}^{(1)}$ resonance from Fig. 4 , for $\theta=0^{\circ},-90^{\circ}$, at a selected temperature, is presented in Fig. 6(a).

This fine splitting shows some weak temperature dependence. The high-temperature interference pattern at $\theta \approx 0^{\circ}$ is strongly distorting the resonant lines. In particular, at high temperatures, where transmission is low, the interference pattern is very irregular and simultaneously strong [Fig. 4(a)], which renders the estimation of the splitting difficult. Therefore we decided to analyze data around $100 \mathrm{~K}$ where the interference pattern is regular for all angles and the resonances can be clearly distinguished from the interference.

We fitted the resonances with functions composed of derivatives of the Lorentzian function and the interference background with a sine function. The properties of the lines determined by this fitting procedure are shown in Fig. 7. Splittings are indeed highest at $\theta=0^{\circ}$, but fits show that they do not close at $\theta= \pm 90^{\circ}$ and stay about $2 \mathrm{GHz}$ apart [Fig. 7(d)]. Therefore, those are rather amplitude-angle dependencies that determine the visibility of fine splittings. The amplitudes of the higher-frequency split-off modes $\left(\Psi_{1}^{(2), 2}\right.$, $\Psi_{1}^{(1), 2}$ ) depend on the angle stronger than those of lowerfrequency split-off modes $\left(\Psi_{1}^{(2), 1}, \Psi_{1}^{(1), 1}\right)$. All four modes have comparable amplitudes around $\theta=0^{\circ}$, but around $\theta= \pm 90^{\circ}$,
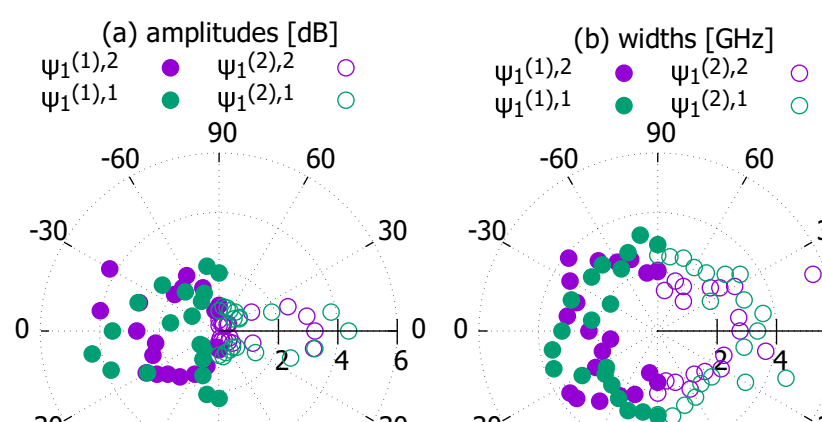

$-60 \quad-60$

$-30$

30

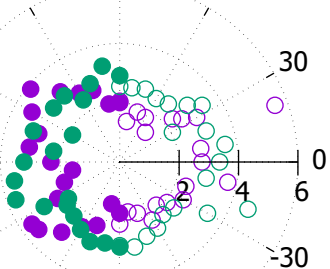

60 $-90$

$-60$

60

$-90-60$

(c) shifted frequencies $[\mathrm{GHz}]$

$\psi_{1}(1)-655 \mathrm{GHz}, \psi_{1}{ }^{(2)}-600 \mathrm{GHz}$

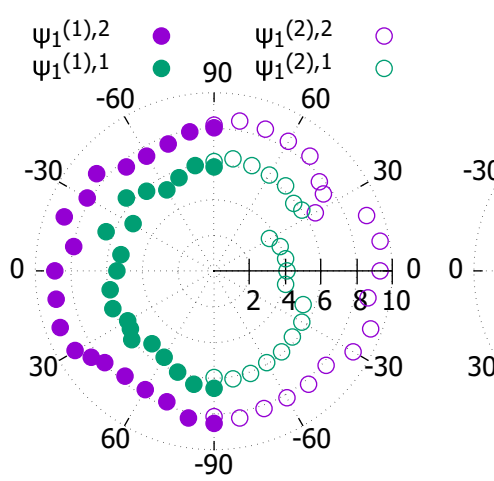

(d) splitting [GHz]

$\begin{array}{lll}\Psi_{1}(1) & 90 & \psi_{1}(2) \\ -60 & & 0\end{array}$

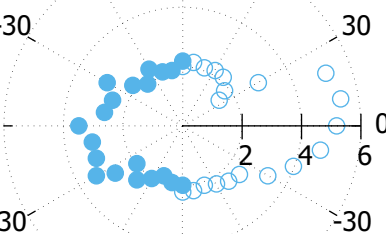

60

$-90$

$-60$

FIG. 7. Angular dependence of parameters of four resonant features in the proximity of $T=100 \mathrm{~K}$. (a) amplitudes, (b) widths, (c) frequencies of resonant features subtracted by offsets labeled in the figure, and (d) fine splitting.

higher-frequency split-off modes are smaller [Fig. 7(a)]. The widths are slightly greater for $|\theta|<45^{\circ}$ [Fig. 7(b)].

The amplitudes of the $\Psi_{1}^{(2)}$ and $\Psi_{1}^{(1)}$ modes show an angular dependence that is in an agreement with those observed in Refs. [28,29,33]. So that the amplitudes are higher for $\mathbf{E}$-field perpendicular to $\mathbf{P}$ and this behavior is much stronger for the $\Psi_{1}^{(2)}$ mode. The fine splitting becomes apparent quite suddenly around $\pm 30^{\circ}$ while rotating the polarization. The symmetry of the fine splitting is identical to that of the dichroism and the birefrigence. This suggest that the observed splitting also originates from the anisotropy of the crystal. However, in the Hamiltonian $[11,32]$, which correctly describes previous angular results [24,29], there is no term which could cause this splitting. Higher-order anisotropy terms, consistent with the rhomohedral symmetry of BFO, are orders of magnitude too small to explain this splitting [43,44]. Neither was such a splitting observed under applied static electric [5] or magnetic [29] fields.

We can estimate the amplitude of an induced polarization $P_{\text {ind }}$, additional to the ferroelectric polarization $\left(P_{F E}=\right.$ $60 \mu \mathrm{C} / \mathrm{cm}^{2}$ ) [1], of an energy equal to that of the observed splitting. This energy is small (about $5 \mathrm{GHz}, 21 \mu \mathrm{eV}$ ), so that we assume that the fine-splitting is caused by a small perturbation to the Hamiltonian. The small value of the fine splitting excludes perturbations of the DzialoshynskiiMoriya interaction or the anisotropy, which are themselves of similar orders of magnitudes as the fine-splitting energy 
( $D_{1}=180 \mu \mathrm{eV}, D_{2}=85 \mu \mathrm{eV}$ and $K=4 \mu \mathrm{eV}$, respectively). Thus, we consider only a perturbation to the much larger exchange interaction $J_{1}=-5.32 \mathrm{meV}$, which is caused by the magnetostriction [45]. The magnetostriction-induced polarization $P_{\text {ind }}$ is estimated as $d J_{1} / d E[45,46]$, where $E$ is an external electric field. Thus, we can estimate the polarization $P_{\text {ind }}$ needed to change $J_{1}$ by the energy equal to that of the fine-splitting $\left(\Delta E_{f s}\right)$, i.e., by solving

$$
\frac{P_{\text {ind }}^{2} V}{2 \epsilon(\omega, \theta) \epsilon_{0}}=\Delta E_{f s},
$$

where $V=372 \times 10^{-30} \mathrm{~m}^{3}$ is the BFO cell volume [1], $\epsilon(\omega, \theta) \approx 30-90$ is the BFO dielectric function and $\epsilon_{0}$ is the vacuum permittivity. This calculation results in $P_{\text {ind }} \approx 1.5 \mathrm{nC} / \mathrm{cm}^{2}$ for the normal ray, which is of the order of the spin-current-induced polarization that leads to the directional dichroism at spin-wave resonances [45]. The $P_{\text {ind }}$ thus found is much smaller than other electric polarizations predicted to be induced by the spin cycloid $[45,46]$.

Thus, we argue that the symmetry of the fine splitting is similar to that of the dielectric function $\epsilon(\omega, \theta)$ and that the only perturbation of the Hamiltonian, large enough to explain the splitting, is caused by magnetostriction [45]. This suggests that the observed effect is related to an interaction of spin waves with phonons as suggested in Ref. [33], an effect that was experimentally observed at phonon frequencies [22,38-40]. Mediators between phonons and spins can be magnetostriction, spin currents or a single-ion anisotropy, which were all discussed in Ref. [45] as leading to an optical activation of the spin-wave modes in BFO by an induced electrical polarization. Excited phonons create an oscillating polarization as expressed in the dielectric function $\epsilon(\omega, \theta)$, which, in birefrigent materials, is a tensor acting on a polarization vector. Therefore, the Hamiltonian, with a perturbation containing the dielectric tensor, might have two solutions for spin-waves modes.

An alternative explanation could be that the spin-wave modes are split by an interaction with photons [47]. This effect would be a function of sample thickness. The angular dependence of the splitting and amplitudes would have to be attributed to the birefrigence and the angular dependence of optical-path length of the extraordinary ray. However, the difference in optical-path lengths is approximately $\left(n_{o}-n_{e}\right) / n_{e} \approx 45 \%$, which cannot account for the observed difference in resonance amplitudes between the normal ray $\left(\theta=0^{\circ}\right)$ and the extraordinay ray $\left(\theta=90^{\circ}\right)$. Furthermore, in our monochromatic experiment, the presence of strongly coupled magnon-polaritons should present itself as clear avoided crossings between Fabry-Perot type cavity modes of the sample and spin-wave modes. We do not observe such a behavior, which should appear every time the resonances cross the interference pattern (Fig. 4).

\section{POLYCRYSTALINE SAMPLES}

We compared the single crystal results with results obtained for a pelletized powder sample in the same experimental setup. Traces of $\Psi_{1}^{(1)}$ mode, presented in Fig. 6, show that the widths of the resonances in the single crystal are narrower than that in the polycrystaline sample. The average over all angles is not sufficient to explain this difference [Fig. 6(c)]. The refractive index of the pelletized sample was determined to be 5.2 at $400 \mathrm{~K}$ and growing about 0.1 per $100 \mathrm{~K}$. The linear extrapolation to $100 \mathrm{~K}$ gives 4.9 , which is about $70 \%$ of the average of the values determined in the current experiment $\frac{1}{3}\left(n_{o}+2 n_{e}\right)=6.9$ at $100 \mathrm{~K}$. This is mostly caused by the porosity of the pellet sample: its density is $64 \%\left(5.5 \mathrm{~g} / \mathrm{cm}^{3}\right)$ of that of the crystal $8.3 \mathrm{~g} / \mathrm{cm}^{3}$, which is the maximum filling by random-packed hard spheres [48]. This comparison of densities of both samples also partially explains why the amplitude of the resonance is smaller in the pelletized sample, even though both the pellet and the crystal samples had similar thicknesses.

\section{CONCLUSIONS}

Dichroism, birefringence and the fine-splitting of spinwave modes, all show the same symmetry: the ordinary ray shows the strongest splitting of spin-wave resonances and lowest transmission at high temperatures; the extraordinary ray shows almost no splitting and much smaller drop of transmission with temperature.

We have shown that (100)-oriented single crystal of bismuth ferrite shows strong dichroism. The lowest transmission is observed for $\mathrm{THz}$ polarization oriented perpendicular to the ferroelectric polarization in the crystal. This dichroism grows exponentially with temperature and reaches about $15 \mathrm{~dB} / \mathrm{mm}$ at room temperature. We attribute this effect as an absorption by $E$ phonon modes. Furthermore, we observed that the crystal is birefringent with $\Delta n \approx-3.0$ and the optical axis is oriented along $\mathbf{P}$. Finally, we have shown the presence of the fine-splittings of the $\Psi_{1}^{(1)}$ and the $\Psi_{1}^{(2)}$ modes, that depend on polarization, with the most pronounced effect for electric field acting perpendicular to the ferroelectric polarization. This symmetry, which is the same as that of the dichroism and the birefrigence, suggest that these splittings result from anisotropic properties of the crystal.

\section{ACKNOWLEDGMENT}

Work partially supported by a Requip (206021 150707/1) grant of the Swiss National Science Foundation and by EPFL.
[1] D. Lebeugle, D. Colson, A. Forget, M. Viret, P. Bonville, J. F. Marucco, and S. Fusil, Phys. Rev. B 76, 024116 (2007).

[2] C. Tabares-Munžoz, J.-P. Rivera, A. Bezinges, A. Monnier, and H. Schmid, Jpn. J. Appl. Phys. 24, 1051 (1985).

[3] T. Zhao, A. Scholl, F. Zavaliche, K. Lee, M. Barry, A. Doran, M. P. Cruz, Y. H. Chu, C. Ederer, N. A. Spaldin, R. R. Das,
D. M. Kim, S. H. Baek, C. B. Eom, and R. Ramesh, Nat. Mater. 5, 823 (2006).

[4] Y.-H. Chu, L. W. Martin, M. B. Holcomb, M. Gajek, S.-J. Han, Q. He, N. Balke, C.-H. Yang, D. Lee, W. Hu, Q. Zhan, P.-L. Yang, A. Fraile-Rodriguez, A. Scholl, S. X. Wang, and R. Ramesh, Nat. Mater. 7, 478 (2008). 
[5] P. Rovillain, R. de Sousa, Y. Gallais, A. Sacuto, M. A. Méasson, D. Colson, A. Forget, M. Bibes, A. Barthélémy, and M. Cazayous, Nat. Mater. 9, 975 (2010).

[6] G. Catalan and J. F. Scott, Adv. Mater. 21, 2463 (2009).

[7] C. Ederer and N. A. Spaldin, Phys. Rev. B 71, 060401 (2005).

[8] I. Sosnowska and A. K. Zvezdin, J. Magn. Magn. Mater. 140, 167 (1995).

[9] I. Sosnowska, T. P. Neumaier, and E. Steichele, J. Phys. C 15, 4835 (1982).

[10] R. S. Fishman, N. Furukawa, J. T. Haraldsen, M. Matsuda, and S. Miyahara, Phys. Rev. B 86, 220402 (2012).

[11] R. S. Fishman, J. T. Haraldsen, N. Furukawa, and S. Miyahara, Phys. Rev. B 87, 134416 (2013).

[12] M. Białek, A. Magrez, A. Murk, and J.-P. Ansermet, Phys. Rev. B 97, 054410 (2018).

[13] J.-P. Rivera and H. Schmid, Ferroelectrics 204, 23 (1997).

[14] C. Jördens, M. Scheller, M. Wichmann, M. Mikulics, K. Wiesauer, and M. Koch, Appl. Opt. 48, 2037 (2009).

[15] Y. Kim, M. Yi, B. G. Kim, and J. Ahn, Appl. Opt. 50, 2906 (2011).

[16] M. Y. Vlasov, V. F. Losev, N. A. Nikolaev, and A. A. Mamrashev, J. Phys.: Conf. Ser. 737, 012022 (2016).

[17] B. T. Thole, G. van der Laan, and G. A. Sawatzky, Phys. Rev. Lett. 55, 2086 (1985).

[18] P. Kuiper, B. G. Searle, P. Rudolf, L. H. Tjeng, and C. T. Chen, Phys. Rev. Lett. 70, 1549 (1993).

[19] S. Polisetty, J. Zhou, J. Karthik, A. R. Damodaran, D. Chen, A. Scholl, L. W. Martin, and M. Holcomb, J. Phys.: Condens. Matter 24, 245902 (2012).

[20] S. Kamba, D. Nuzhnyy, M. Savinov, J. Šebek, J. Petzelt, J. Prokleška, R. Haumont, and J. Kreisel, Phys. Rev. B 75, 024403 (2007).

[21] J. Petzelt and S. Kamba, Ferroelectrics 503, 19 (2016).

[22] R. P. S. M. Lobo, R. L. Moreira, D. Lebeugle, and D. Colson, Phys. Rev. B 76, 172105 (2007).

[23] I. Kézsmárki, U. Nagel, S. Bordács, R. S. Fishman, J. H. Lee, H. T. Yi, S.-W. Cheong, and T. Rõõm, Phys. Rev. Lett. 115, 127203 (2015).

[24] D. Talbayev, S. Lee, S.-W. Cheong, and A. J. Taylor, Appl. Phys. Lett. 93, 212906 (2008).

[25] M. Cazayous, Y. Gallais, A. Sacuto, R. de Sousa, D. Lebeugle, and D. Colson, Phys. Rev. Lett. 101, 037601 (2008).

[26] M. K. Singh, R. S. Katiyar, and J. F. Scott, J. Phys.: Condens. Matter 20, 252203 (2008).

[27] A. Kumar, J. F. Scott, and R. S. Katiyar, Appl. Phys. Lett. 99, 062504 (2011).
[28] D. Talbayev, S. A. Trugman, S. Lee, H. T. Yi, S.-W. Cheong, and A. J. Taylor, Phys. Rev. B 83, 094403 (2011).

[29] U. Nagel, R. S. Fishman, T. Katuwal, H. Engelkamp, D. Talbayev, H. T. Yi, S.-W. Cheong, and T. Rõõm, Phys. Rev. Lett. 110, 257201 (2013).

[30] J. Buhot, C. Toulouse, Y. Gallais, A. Sacuto, R. de Sousa, D. Wang, L. Bellaiche, M. Bibes, A. Barthélémy, A. Forget, D. Colson, M. Cazayous, and M.-A. Measson, Phys. Rev. Lett. 115, 267204 (2015).

[31] S. Skiadopoulou, V. Goian, C. Kadlec, F. Kadlec, X. F. Bai, I. C. Infante, B. Dkhil, C. Adamo, D. G. Schlom, and S. Kamba, Phys. Rev. B 91, 174108 (2015).

[32] R. S. Fishman, Phys. Rev. B 87, 224419 (2013).

[33] E. Matsubara, T. Mochizuki, M. Nagai, and M. Ashida, Phys. Rev. B 94, 054426 (2016).

[34] T. Ito, T. Ushiyama, Y. Yanagisawa, R. Kumai, and Y. Tomioka, J. Cryst. Growth 11, 5139 (2011).

[35] C. Caspers, V. P. Gandhi, A. Magrez, E. de Rijk, and J.-P. Ansermet, Appl. Phys. Lett. 108, 241109 (2016).

[36] P. Nemec, M. Fiebig, T. Kampfrath, and A. V. Kimel, Nat. Phys. 14, 229 (2018).

[37] M. K. Singh, S. Ryu, and H. M. Jang, Phys. Rev. B 72, 132101 (2005).

[38] R. Haumont, J. Kreisel, P. Bouvier, and F. Hippert, Phys. Rev. B 73, 132101 (2006).

[39] H. Fukumura, H. Harima, K. Kisoda, M. Tamada, Y. Noguchi, and M. Miyayama, J. Magn. Magn. Mater. 310, e367 (2007).

[40] P. Rovillain, M. Cazayous, Y. Gallais, A. Sacuto, R. P. S. M. Lobo, D. Lebeugle, and D. Colson, Phys. Rev. B 79, 180411 (2009).

[41] M. Avendaño-Alejo, Opt. Express 13, 2549 (2005).

[42] G. A. Komandin, V. I. Torgashev, A. A. Volkov, O. E. Porodinkov, I. E. Spektor, and A. A. Bush, Phys. Solid State 52, 734 (2010).

[43] R. Fishman, Physica B 536, 115 (2018).

[44] R. S. Fishman, Phys. Rev. B 97, 014405 (2018).

[45] R. S. Fishman, J. H. Lee, S. Bordács, I. Kézsmárki, U. Nagel, and T. Rõõm, Phys. Rev. B 92, 094422 (2015).

[46] J. H. Lee and R. S. Fishman, Phys. Rev. Lett. 115, 207203 (2015).

[47] K. Grishunin, T. Huisman, G. Li, E. Mishina, T. Rasing, A. V. Kimel, K. Zhang, Z. Jin, S. Cao, W. Ren, G.-H. Ma, and R. V. Mikhaylovskiy, ACS Photon. 5, 1375 (2018).

[48] C. Song, P. Wang, and H. A. Makse, Nature (London) 453, 629 (2008). 